\title{
Design-Based Texture Feature Fusion Using Gabor Filters and Co-Occurrence Probabilities
}

\author{
David A. Clausi and Huang Deng
}

\begin{abstract}
A design-based method to fuse Gabor filter and grey level co-occurrence probability (GLCP) features for improved texture recognition is presented. The fused feature set utilizes both the Gabor filter's capability of accurately capturing lower and mid-frequency texture information and the GLCP's capability in texture information relevant to higher frequency components. Evaluation methods include comparing feature space separability and comparing image segmentation classification rates. The fused feature sets are demonstrated to produce higher feature space separations, as well as higher segmentation accuracies relative to the individual feature sets. Fused feature sets also outperform individual feature sets for noisy images, across different noise magnitudes. The curse of dimensionality is demonstrated not to affect segmentation using the proposed the 48-dimensional fused feature set. Gabor magnitude responses produce higher segmentation accuracies than linearly normalized Gabor magnitude responses. Feature reduction using principal component analysis is acceptable for maintaining the segmentation performance, but feature reduction using the feature contrast method dramatically reduced the segmentation accuracy. Overall, the designed fused feature set is advocated as a means for improving texture segmentation performance.
\end{abstract}

Index Terms-Brodatz, clustering, feature contrast (FC), Fisher linear discriminant (FLD), grey level co-occurrence matrix, grey level co-occurrence probability (GLCP), K-means, principal component analysis (PCA), segmentation, texture analysis.

\section{INTRODUCTION}

$\mathbf{T}$ EXTURE segmentation is the task of identifying regions with similar patterns in an image. There is no known method that is able to consistently and accurately segment textured images. A commonly used strategy for texture segmentation is to first extract features on a pixel-by-pixel basis from an image and then use some technique to classify the extracted features [1]-[3]. To improve the overall quality of image texture segmentation, either the quality of the texture features or the quality of the classification algorithm must be improved. This paper focuses on the improvement of the quality of the texture features and the preliminary work published in [4] acted as a basis for this research.

Manuscript received December 22, 2003; revised November 4, 2004. This work was supported in part by CRYSYS (CRYospheric SYStem in Canada, http://www.crysys.ca) and the NSERC (Natural Sciences and Engineering Research Council, http://www.nserc.ca) NCE-sponsored (Networks of Centers of Excellence) program GEOIDE (Geomatics for Informed Decisions, http://www.geoide.ulaval.ca). The associate editor coordinating the review of this manuscript and approving it for publication was Dr. Eli Saber.

D. A. Clausi is with the Department of Systems Design Engineering, University of Waterloo, Waterloo, ON N2L 3G1 Canada (e-mail: dclausi@engmail.uwaterloo.ca).

H. Deng is with PCI Geomatics, Gatineau, QC J8Y 3 Y7 Canada (e-mail: deng@pcigeomatics.com).

Digital Object Identifier 10.1109/TIP.2005.849319
There exist numerous methods for performing texture feature extraction. Tuceryan and Jain [5] divide these methods into four categories: statistical, geometrical, model-based, and signal processing methods. Many papers exist that focus on the analysis or development of individual texture feature methods. However, a pair of drawbacks are noted in the research literature. First, few papers consider the fusion of texture features. Proper fusion or combining of features derived from different texture methods is expected to produce an improved feature set. Second, few papers consider the comparison of existing texture feature methods, especially for the specific application of image texture segmentation. Papers presenting such comparisons tend to look at the classification problem, where pure fixed sized texture samples are used for training and testing, instead of the more complex problem of segmentation which must account for texture boundaries where windowed samples contain two or more distinct textures. By directly comparing different feature extraction techniques, relative abilities can be quantitatively determined.

A perceived drawback of feature fusion is the "curse of dimensionality" [6]. This concept recognizes that there is a limit to the feature space dimension to produce maximum accuracy, given a finite number of feature vectors for a particular class. Once the number of features exceeds a certain limit, the classification accuracy begins to decrease. To address this potential problem, scientists motivate feature reduction techniques [7]-[9].

This paper next considers the background literature on each of the above topics (Section II). Section III discusses the use of two popular texture feature extraction methods [grey level co-occurrence probabilities (GLCPs) and Gabor filters] and their design-based fusion methodology. Evaluation of the fused feature set is performed using discriminant analysis and segmentation testing. Specific concepts such as the role of the curse of dimensionality, image noise, linear normalization of features, and feature space reduction are also investigated with respect to the proposed fused feature set.

\section{BACKGROUND}

\section{A. Texture Feature Fusion}

Fusion is a broad-based term that is used in a variety of scientific settings. Texture features have been fused with other features for applications such as face recognition [10] and image segmentation [11]. Here, the focus is on a methodology to combine or fuse features produced using different texture extraction methods. There exists limited research fusing texture features derived from different methods. These examples often fuse by 
concatenation of different feature sets. Solberg and Jain [8] performed a supervised classification of four satellite-based synthetic aperture radar (SAR) images using a variety of texture methods and noted that a feature fusion improved the classification rate. Bashar and Ohnishi [12] fuse texture features based on various texture features by concatenation as well as correlation. Chindaro et al. [13] fuse texture features from different color spaces. Trianni et al. [14] fuse different texture measures and then perform a feature selection to investigate texture segmentation of urban scenes. Zongker [15] evaluated different feature reduction methods on a fused set of texture features. Clausi [16] presented a detailed analysis fusing features based on GLCPs, Gabor filters, and Markov random fields (MRFs) for classifying SAR sea ice imagery. Relatively uncorrelated feature sets produced statistically significant improvements when concatenated.

Here, the focus is on a methodology to combine or fuse features produced using different texture extraction methods. There exists limited research on the topic of fusing features derived from different texture methods. In all of the cases in the above paragraph, texture feature fusion is performed blindly by concatenating the feature sets without any basis. In this paper, a design rationale is provided for supporting fusion of certain texture features. Such a design process has not been, to the best of the authors' knowledge, represented in the research literature.

\section{B. Feature Reduction and the Curse of Dimensionality}

Feature fusion can lead to an increase in feature space dimensionality. For a fixed number of samples, increasing the feature space dimension will eventually cause the classification accuracy to decline. This is known as the "curse of dimensionality" (or, in other terms, the "curse of finite sample size") [6]. So, if $n$ feature vectors are required to estimate a parameter (such as the mean or variance) for a one-dimensional feature space, from a theoretical perspective, $n^{N}$ features would be required for the $\mathrm{N}$-d feature space. However, in practice, linear approximations are acceptable (i.e., $n N$ features). Jain and Chandrasekaran [6] state "The general guidelines for having five to ten times as many samples as measurements still seems to be a good practice to follow" (p. 852). Another reason to perform feature reduction is to minimize the number of calculations in the subsequent classification procedure.

Pichler et al. [7] recognize that the curse of dimensionality will reduce segmentation performance when the optimum number of features is exceeded. To reduce the feature dimension produced by a Gabor filter bank, they develop their "feature contrast" (here, referred to as "FC") method. Solberg and Jain [8] noted that classification performance was reduced if they used all of their 19 texture features. For each of four different SAR remote-sensing scenes, a set of ten different features were produced that generated the preferred classification. Bigun [9] applies principal component analysis (PCA) to their feature images both globally and locally to reduce the feature space dimensionality. They comment that applying the PCA transform to the entire feature set can be potentially problematic due to the undesired interaction between classes. Jain and Farrokhnia [3] utilize a feature selection scheme using a least squares error criterion. The method is applied to features produced by a Gabor filter bank.

\section{Feature Space Normalization}

Normalization (scaling) is used in pattern recognition so that certain features (perhaps with larger range or variance) do not dominate the distance calculations during classification [17]. The normalization should allow each feature component to be treated equally with respect to its contribution to the distance. As the feature vectors for segmentation are spread due to the presence of subclasses, it can be quite inappropriate to normalize the feature vector to be of zero mean and unit variance [18]. A common method to normalize texture features is to individually scale each feature dimension of the entire feature set to the range $[0,1][17]$. The methodology of the normalization is quite important to the overall success of the texture segmentation, yet this is a topic that is not known to be comparatively tested in texture segmentation research.

\section{Texture Feature Comparisons}

There exist various papers that compare texture feature methods in the context of supervised classification (i.e., use of separate training and test subimages containing pure texture regions). Examples include an early study by Weszka et al. [19], a theoretical perspective by Conners and Harlow [20], an examination of multiple texture methods by Ohanian and Dubes [21], and studies using remotely sensed data by Clausi [16] and Barber et al. [22]. The primary purpose of this paper is to investigate the more difficult problem of unsupervised texture segmentation, where no training data is used and texture feature vectors are often derived from image regions that contain a mixture of two or more textures. The literature on comparison of different texture methods for unsupervised texture segmentation is limited compared to supervised texture segmentation. Three comparative texture segmentation papers (one supervised, two unsupervised) are presented here.

Clausi and Yue [23] consider the role of GLCPs and MRFs for the purpose of unsupervised texture segmentation of SAR sea ice imagery. The role of window size in texture feature separability as well as the role of multiple textures within a window are investigated. GLCPs are demonstrated to have improved discrimination ability relative to MRFs with decreasing window size. On the other hand, GLCPs are more sensitive to texture boundary confusion than MRFs. Du Buf et al. [24] perform an unsupervised segmentation study of primarily bipartite images. From seven feature extraction methods, the GLCP features are identified as one of the three preferred methods. Randen and Husoy [2] compare an exhaustive list of signal processing techniques for the purpose of supervised image texture segmentation. They compare these methods to two classical methods: GLCPs and autoregressive (model-based) approaches. The test results did not produce any definitive recommendations.

\section{TEXTURE FEATURE FUSION}

In this section, the two texture methods will each be briefly described. Then, a design method for feature fusion will be 
presented. The fused feature set will serve as a basis for the subsequent testing.

\section{A. Texture Feature Extraction Methods}

1) Gabor Filters: Papers that fully describe the use of Gabor filters for texture segmentation include [3], [25], and [26]. For texture analysis, Gabor filters have the ability to model the frequency and orientation sensitivity characteristic of the human visual system. A Gabor function is a Gaussian modulated complex sinusoid in the spatial domain. The two-dimensional Gaussian has an aspect ratio of $\sigma_{x} / \sigma_{y}$. The complex exponential has a spatial frequency of $F$ and an orientation $\theta$. The mathematical tractability of the filter in the spatial-frequency domain is appealing since it is simply a Gaussian centered on the frequency of interest [26], e.g.

$$
H(u, v)=\exp \left[-2 \pi^{2}\left\{(u-F)^{2} \sigma_{x}^{2}+v^{2} \sigma_{y}^{2}\right\}\right] .
$$

Typically, a filter configuration that allows for complete coverage of the spatial-frequency plane is created. The filters are set up in a pseudowavelet format to match the filter's frequency with its spatial extent. Each pixel will have a response to each filter, so each pixel is represented by a feature vector dimensioned to the number of filters.

2) GLCPs: The grey level co-occurrence texture method proposed by Haralick et al. [27] is a common method for texture feature extraction [16], [28]-[30]. The first step is to determine co-occurring probabilities of all pairwise combinations of quantized $(G)$ grey levels $(i, j)$ in the fixed-size $(N)$ spatial window given two parameters: inter-pixel distance $(\delta)$ and orientation $(\theta)$, i.e.

$$
P(i, j)=\operatorname{Pr}(i, j \mid \delta, \theta, G, N) .
$$

Usually, a variety of orientations and inter-pixel distances are selected. Coarser quantization $(G)$ can accelerate calculations and reduce noise but also lose texture information [28], [29]. The ability to capture texture features is determined by window size $N$. Small windows can lead to poor local estimates and large windows increase the risk of multiple textures appearing in the window which produces misleading features [23].

The second step is to apply statistics to the co-occurring probabilities. Statistics that identify some structural aspect of the arrangement of the co-occurring probabilities (which, in turn, reflect some qualitative characteristic of the local image texture, e.g., smoothness or roughness) are applied to generate the texture features. Each window generates a feature vector which is associated with the center pixel of the window. As a result, each pixel in the image (excluding border regions) has a feature vector associated with it.

\section{B. Parameter Selection}

1) Gabor Filter Parameter Selection: Gabor filters are perfectly adept at characterizing pure sinusoid signals, regardless of the frequency. Given that any practical signal can be decomposed into a weighted sum of sinusoids, Gabor filters are well-suited to decomposing textural information. A tuned Gabor filter applied to a sinusoid will generate a flat magnitude response. This is obviously desirable for pattern recognition, so that a consistent feature measurement is produced [25]. In the presence of point noise, Gabor filters are able to generate consistent measurements in low and medium frequencies but generate inconsistent measurements for higher frequencies. The higher the frequency of a Gabor filter, the more sensitive the filter is to the point noise. This is due to the higher frequency filters having larger spatial-frequency bandwidth which covers relatively more energy of the impulsive noise that is evenly distributed in the spatial-frequency domain. High frequency Gabor filters created from a pseudowavelet filter bank implementation have very short duration with only a few significant weights. Such weights are more noise susceptible than the low and midfrequency filters.

An example of the impact of additive white noise on the feature extraction ability of Gabor filters is presented. Long duration unit sinusoids with periodicity ranging from 2.0 to 25.0 pixels per cycle (ppc) [equivalently, frequencies of 0.04 to 0.5 cycles per pixel (cpp)] were created and zero mean Gaussian noise $(\sigma=0.2)$ was added to each signal. A complex Gabor filter with a matched frequency was convolved with each signal and the magnitude response determined. Let $\sigma$ and $\mu$ denote the standard deviation and mean of the magnitude response. The ratio $\sigma / \mu$ reflects the variation of magnitude response over the entire signal. The value of $\sigma / \mu$ for the magnitude response was determined for each frequency and plotted in Fig. 1. With higher local frequencies (note that this corresponds to decreasing ppc), $\sigma / \mu$ increases indicating that the Gabor filter estimates of natural data are not expected to be accurate for signal components containing high frequencies.

2) GLCP Parameter Selection: To replace the high-frequency Gabor filter features with some other more suitable features is appropriate. The GLCP features are able to play such a role. If the inter-pixel distance $\delta$ is set to 1 or 2 , the corresponding GLCP features measure local high frequency information. The GLCP method assumes a uniform distribution across the window of interest as opposed to Gabor filters which use a Gaussian weighting. As a result, the GLCP method is expected to be not as susceptible to local additive noise. The same sinusoidal signals used for Gabor filter testing are used for testing GLCP features. The selected GLCP statistics are contrast, entropy, and correlation. Each feature is retrieved from both the noise-free sinusoidal signal and the corresponding noisy signal. The $\sigma / \mu$ for entropy is plotted in Fig. 2. This plot is similar to those produced using correlation and contrast and these indicate that the GLCP features experience the same variability across all frequencies.

\section{Feature Fusion}

1) Fusion Implementation: The results in the previous subsection indicate that substituting GLCP texture features for the high frequency Gabor filters is an appropriate design strategy for texture feature fusion. If GLCPs are preferred to Gabor filters for detecting higher frequency signals, why not use this method exclusively for texture feature extraction? There are numerous reasons for not doing this.

To substitute GLCP features for the low and mid-frequency Gabor filters for unsupervised applications is inappropriate. 


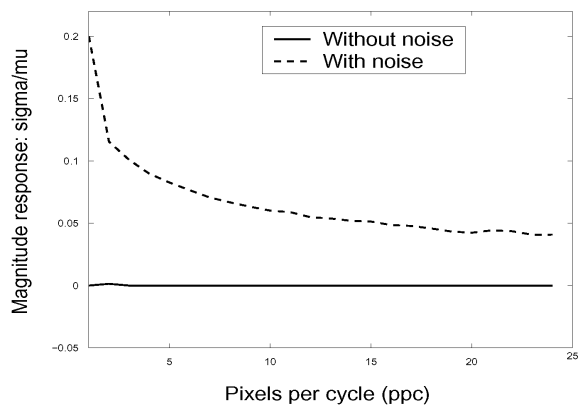

Fig. 1. Ratio of Gabor filter magnitude response $\sigma$ to $\mu$ as a function of signal periodicity (ppc) for sinusoids with additive point noise.

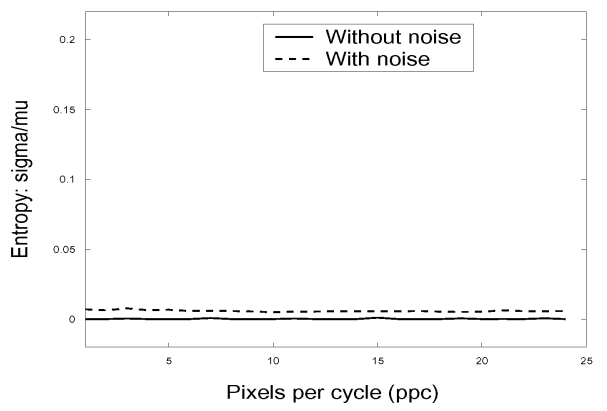

Fig. 2. Ratio $\sigma / \mu$ for GLCP features produced using entropy. Contrast and correlation statistics produce similar results.

The GLCP method requires many parameters be set. To exhaustively select the quantization, window size, and displacements for all possible cases (to capture the low and mid-frequency components) is computationally prohibitive. There is no known method to automatically select these parameters to generate a preferred texture feature set. Zucker and Terzopoulos [31] developed a supervised technique to select these parameters which still requires exceptional computational demands to determine GLCPs. In addition, in-house testing has revealed that there is a high degree of correlation for preferred GLCP features (using [31]) and matched Gabor filters, negating the need to calculate low and mid-frequency GLCP texture features. Also, to collect low and mid-frequency texture features using the GLCP method would involve increasing the window size; however, these larger window sizes will capture information with respect to many frequencies, not just the lower frequency information. In the Gabor pseudowavelet filter bank, filters with larger spatial bandwidth are automatically associated only with lower frequency signals. For these reasons, the GLCP texture features are not recommended to be used exclusively for unsupervised texture segmentation.

2) Gabor Parameters Used: An experimentally supported preferred Gabor feature set is used [25]. A total of 24 complex Gabor filters at four frequencies $(22.63,11.31,5.66$, and $2.83 \mathrm{ppc})$ and six orientations $\left(0^{\circ}, 30^{\circ}, 60^{\circ}, 90^{\circ}, 120^{\circ}\right.$, and $150^{\circ}$ ) are chosen to filter each test image. Although there exist many techniques to extract features from Gabor filter outputs, there is experimental evidence by Clausi and Jernigan [25] to support using the magnitude response. As per Bovik et al. [26], the magnitude image corresponding to each filter will be smoothed using a scaled Gaussian (the scale is 2/3). A 24-dimensional (24-D) Gabor filter feature vector can be obtained
TABLE I

Denotations of TeXture FeAture SETs

\begin{tabular}{l|l}
\hline$F_{G 18}$ & $\begin{array}{l}\text { The feature set using the } 18 \text { Gabor filter features } \\
\text { at the three lowest frequencies. } \\
F_{G 24}\end{array}$ \\
$F_{C 24}$ & $\begin{array}{l}\text { The feature set using all } 24 \text { Gabor filter features. } \\
\text { The feature set using all } 24 \text { co-occurrence }\end{array}$ \\
$F_{G 18}^{C 24}$ & The feature set fusing $F_{G 18}$ and $F_{C 24}$. \\
$F_{G 24}^{C 24}$ & The feature set fusing $F_{G 24}$ and $F_{C 24}$. \\
\hline
\end{tabular}

for each pixel. The feature set excluding the highest frequency is denoted by $F_{G 18}$, and the feature set using all 24 Gabor filter features is denoted by $F_{G 24}$ (see Table I).

3) GLCP Parameters Used: There are many GLCP statistics that can be used; however, only three statistics are advocated [28] (contrast, entropy, and correlation) and will be used here. A $9 \times 9$ window is used. By choosing two inter-pixel distances $(\delta=1, \delta=2)$ and four orientations $\left(0^{\circ}, 45^{\circ}, 90^{\circ}\right.$, and $\left.135^{\circ}\right)$, a total of eight sets of GLCPs are determined. A quantization level of 64 is set to quantize each image texture [16], [29]. Thus, each pixel is represented by a 24-D co-occurrence probability feature vector, denoted by $F_{C 24}$ (see Table I).

4) Gabor and GLCP Fusion: The GLCP texture features produce more consistent measurements at high signal frequencies than the tuned Gabor filters. As a result, the GLCP features can be used to substitute for or supplement the Gabor high frequency band. To substitute for the high frequency band, $F_{G 18}$ can be combined with $F_{C 24}$. This fused feature set is denoted by $F_{G 18}^{C 24}$. To supplement, $F_{G 24}$ can be fused with $F_{C 24}$. This fused feature set is denoted by $F_{G 24}^{C 24}$. Table I represents those feature sets that will be compared in the subsequent testing.

\section{Discriminant Analysis Testing AND Results}

To evaluate the quality of the texture feature sets, both discriminant and segmentation testing are performed. In this section, the Fisher linear discriminant (FLD) is used to measure and compare the separability of the Gabor and GLCP texture features, as well as their fused sets and feature space reductions. In the next section (Section V), segmentation testing is performed.

\section{A. Discriminant Analysis Hypotheses}

Hypothesis 1: The fused feature sets $\left(F_{G 24}^{C 24}, F_{G 18}^{C 24}\right)$ will have higher feature space separability between classes compared to the individual feature sets $\left(F_{G 24}, F_{G 18}, F_{C 24}\right)$.

Hypothesis 2: Feature reduction is expected to erode the class feature space separability.

\section{B. Test Images}

A total of thirteen Brodatz [32] image textures are selected for discriminant analysis. The names of the textures and their 
TABLE II

Class Pairwise Values of $\tau$ FOR Fused Feature Set $F_{G 24}^{C 24}$ FOR Brodatz Classes

\begin{tabular}{|c|c|c|c|c|c|c|c|c|c|c|c|c|c|}
\hline & D002 & D006 & D021 & D034 & D038 & D052 & D055 & D057 & D068 & D076 & D077 & D078 & D104 \\
\hline D002 & - & 123.99 & 300.49 & 158.65 & 76.41 & 107.06 & 73.14 & 54.57 & 95.85 & 74.03 & 199.10 & 96.44 & 113.63 \\
\hline D006 & - & - & 342.79 & 305.59 & 172.96 & 257.68 & 258.33 & 133.05 & 90.31 & 111.26 & 317.85 & 188.87 & 246.10 \\
\hline D021 & - & - & - & 455.67 & 245.67 & 457.59 & 253.30 & 189.03 & 363.95 & 235.53 & 375.05 & 234.69 & 143.74 \\
\hline D034 & - & - & - & - & 75.11 & 303.81 & 150.11 & 101.05 & 194.87 & 149.96 & 385.64 & 204.23 & 265.33 \\
\hline D038 & - & - & - & - & - & 113.74 & 57.26 & 62.06 & 133.18 & 102.55 & 248.50 & 62.58 & 187.79 \\
\hline D052 & - & - & - & - & - & - & 136.69 & 56.45 & 204.74 & 181.27 & 322.82 & 162.00 & 184.93 \\
\hline D055 & - & - & - & - & - & - & - & 62.81 & 151.42 & 91.22 & 145.34 & 90.00 & 158.63 \\
\hline D057 & - & - & - & - & - & - & - & - & 109.03 & 88.17 & 154.42 & 82.44 & 92.57 \\
\hline D068 & - & - & - & - & - & - & - & - & - & 46.55 & 239.44 & 39.35 & 150.79 \\
\hline D076 & - & - & - & - & - & - & - & - & - & - & 161.55 & 56.50 & 94.19 \\
\hline D077 & - & - & - & - & - & - & - & - & - & - & - & 147.41 & 241.06 \\
\hline D078 & - & - & - & - & - & - & - & - & - & - & - & - & 198.96 \\
\hline D104 & - & - & - & - & - & - & - & - & - & - & - & - & - \\
\hline
\end{tabular}

associated Brodatz numbers are: field stone (D002), wire (D006), canvas (D021), netting (D034), water (D038), shaw cloth (D052), straw matting (D055), paper (D057), wood grain (D068), fiber cloth (D076), cotton canvas (D077), straw cloth (D078), and loose burlap (D104). A $256 \times 256$-size image for each texture is used to extract features. Only the features for the central $128 \times 128$-pixel region are used to avoid boundary influences. Feature vectors for each image were created for each feature set identified in Table I.

\section{Hypothesis 1: Discriminant Analysis of Fused Feature Sets}

This hypothesis states that the fused feature sets $\left(F_{G 24}^{C 24}\right.$, $F_{G 18}^{C 24}$ ) will have higher feature space separability between classes compared to the individual feature sets $\left(F_{G 24}\right.$, $\left.F_{G 18}, F_{C 24}\right)$. The FLD [17] is used since it is a recognized nonparametric method to analyze the class separation in the feature space. The FLD is determined by calculating the Fisher criterion $\tau(\omega)=\tau=\omega^{T} S_{B} \omega / \omega^{T} S_{W} \omega$, where $S_{B}$ and $S_{W}$ are the between-class and within-class scatter matrices. Given space constraints, only the feature set with the best separability $\left(F_{G 24}^{C 24}\right)$ has all of its class-pairwise $\tau$ values presented here (Table II).

Each of the other feature sets had an average $\tau$ less than that obtained by the feature set $F_{G 24}^{C 24}$. The average $\tau$ (averaged over all possible class pairs) and the ratio of this average to the average for $F_{G 24}^{C 24}$ are reported (Table III). Clearly, the fused feature sets have stronger separability than any of the individual feature sets. $F_{G 24}$ and $F_{G 18}$ each have significantly higher separability than the GLCP set $F_{C 24}$ (with a ratio of 0.137).

\section{Hypothesis 2: Impact of Feature Reduction on Discriminant} Analysis

This hypothesis states that the feature reduction is expected to erode the class feature space separability. The impact of feature reduction on the measured class separability is usually not assessed in other publications in the research literature. Here, PCA [17] and the feature contrast (FC) method [7] are applied
TABLE III

Class Feature Space Separability for All Nine Texture Feature Sets By AVERAGE Class-PairWise Fisher LinEar CRITERION VALUES.

The Ratio of Each Feature SET'S Average $\tau$ Relative to THE $\tau$ FOR THE Most Separable Feature Set $F_{G 24}^{C 24}$ Is Also Presented

\begin{tabular}{|c|c|c|}
\hline Feature Set & Average $\left(\tau_{a v g}\right)$ & Ratio to $\tau_{a v g}$ for $F_{G 24}^{C 24}$ \\
\hline$F_{G 24}^{C 24}$ & 173.09 & 1 \\
\hline$F_{G 18}^{C 24}$ & 163.69 & 0.946 \\
\hline$F_{G 24}$ & 113.94 & 0.658 \\
\hline$F_{G 18}$ & 106.74 & 0.617 \\
\hline$F_{C 24}$ & 23.74 & 0.137 \\
\hline$F_{G 24}^{C 24}$ by PCA $(98 \%)$ & 141.37 & 0.817 \\
\hline$F_{G 24}^{C 24}$ by $\mathrm{FC}(5 \%)$ & 61.01 & 0.353 \\
\hline$F_{G 18}^{C 24}$ by PCA $(98 \%)$ & 136.91 & 0.791 \\
\hline$F_{G 18}^{C 24}$ by $\mathrm{FC}(5 \%)$ & 57.18 & 0.330 \\
\hline
\end{tabular}

to the two fused feature sets. For PCA, the number of features was reduced to retain $98 \%$ of the total energy. The FC method followed the methodology used by Pichler et al. [7]. In summary, the feature contrast value $\left(C_{i}\right)$ for each feature dimension (i)) is calculated by

$$
C_{i}=\frac{\max \left(f_{i}\right)-\operatorname{mean}\left(f_{i}\right)}{\max \left(f_{i}\right)+\operatorname{mean}\left(f_{i}\right)}
$$

where $\max \left(f_{i}\right)$ and mean $\left(f_{i}\right)$ represent the maximum and mean feature value for the $i$ th feature dimension (across all classes). Then, the feature values in each dimension are multiplied by the weight:

$$
W_{i}=\left(\frac{C_{i}}{C_{\max }}\right)^{n}
$$


where $C_{\max }$ represents the largest of the $C_{i}$ values. The FC implementation here follows the methodology advocated by Pichler et al.; namely, the weights are applied to normalized feature vectors, the Gabor features are represented in power, only those $W_{i}$ that exceed 5\% are retained, and $n$ is set to 4 .

These results, represented in Table III, indicate that both methods used for feature reduction cause reductions in feature space separability. For example, for both fused feature sets $F_{G 24}^{C 24}$ and $F_{G 18}^{C 24}$, PCA causes the average separability to be reduced by approximately $20 \%$. The number of components retained by PCA is 34 for $F_{G 24}^{C 24}$ and 30 for $F_{G 18}^{C 24}$. The variability removed by the PCA method seems important for class separability in the feature space. The FC method reduced the separability of these fused feature sets by approximately two thirds. Note that the FC method was originally used by Pichler et al. to reduce only Gabor filter outputs; however, for the case of these thirteen textures, no $W_{i}$ for $F_{G 24}$ had values below 5\% and, since FC is a linear transformation, no changes to the FLD separability of the Gabor filtered feature sets occurred. The number of feature dimensions retained by $\mathrm{FC}$ is 32 for $F_{G 24}^{C 24}$ and 26 for $F_{G 18}^{C 24}$. An explanation for the dramatic reduction in separability using the FC method is provided under Hypothesis 7. The results for the first two hypotheses imply that the fused feature sets should produce higher segmentation accuracies and that the feature reduction should decrease segmentation performance.

\section{Segmentation Testing}

In this section, various segmentation-based hypotheses are tested with regards to the nonfused and fused texture feature sets presented in Table I.

\section{A. Segmentation Hypotheses}

Hypothesis 3: The curse of dimensionality will not reduce segmentation accuracies when using the 48-dimensional (48-D) feature set.

Hypothesis 4: The fused feature sets will achieve higher segmentation accuracies than using any nonfused set.

Hypothesis 5: In the presence of noise, the fused feature set will achieve higher segmentation accuracies than using nonfused feature sets.

Hypothesis 6: Linear normalization of pure Gabor filtered texture features will reduce their segmentation ability.

Hypothesis 7: Feature reduction of fused texture feature sets will lead to poorer segmentation ability.

\section{B. Experimental Methods}

1) Clustering: Given that there is no absolute best method to cluster the texture features, the standard K-means method [17] is used to cluster the feature vectors and generate a segmented image. Also, K-means is an unsupervised approach which is well-suited to identifying a limited number of distinct clusters, which is expected when segmenting textured images.

2) Feature Normalization: As the K-means clustering method is implemented according to the criterion of minimizing the Euclidian distance between feature vectors, normalizing the feature vectors is generally necessary. Here, linear normalization (scaling each feature dimension independently to a range of $(0,1)[17])$ is applied to all feature sets, except for certain cases in Hypothesis 6 where no normalization is used.

3) Test Images: Three Brodatz mosaic images are used for testing. Fig. 4(a) illustrates the image originally published by Bigun and Du Buf [33]. Seven Brodatz textures (D021, D002, D052, D068, D076, D034, and D006, introduced in raster order) are combined in a sixteen patch mosaic so that each texture's boundary touches each of the others at least once. One texture is noticeably complex (D002) and the rest have regular patterns with varying spatial frequencies. Horizontal boundaries are straight, but the vertical boundaries are generated by a random walk procedure. In this paper, the image is referred to as the "Bigun" image. The second image (Fig. 5), referred to as the "Patch" image, contains five textures (D038, D057, D077, D078, and D104) in six patches. Each Brodatz texture is represented by a different number of pixels and all of the texture boundaries are straight. The third image (Fig. 6, referred to as the "Star" image) has texture boundaries that are considerably more complex. Five Brodatz texture classes are included (D038, D055, D057, D077, and D078) in irregular shaped patches to capture how different boundary shapes may affect the final segmentation results. Note that the 15-pixel boundary for each image is removed from each texture feature set to reduce the influence of the image boundary.

4) Reporting Accuracies: The segmentation accuracy (percentage) for all testing is presented in Table IV. Here, the average of $25 \mathrm{~K}$-means runs is reported for each test to provide an understanding of the expected classification ability under operational conditions (when the true segmentation is not known). The same set of random seeds is used for each feature set.

Although reporting classification accuracies is insightful, it is recognized that this alone is not ideal for comparing different results. During testing, it was noted that an accurate segmentation often meant that each texture in the image was uniquely identified. When a segmentation procedure broke down, often two classes were merged as the same cluster and a texture border (usually in a high contrast region) was erroneously identified as a separate class. Mao and Jain also noted this failure mode [18]. One could assume that a successful segmentation would occur if the classes were uniquely identified (i.e., no two classes were merged as the same cluster and no boundary between two distinct textures was identified as a separate cluster; however, the segmentation may still have some minor errors in both interior and boundary regions). So, in addition to percentage accuracies, total counts of successful segmentations across $25 \mathrm{~K}$-means runs (i.e., when all classes are uniquely identified) are provided. This process ignores how accurately each class is identified, but only cares whether or not all the classes are uniquely identified. This count is referred to as the all-class identification count or the AIC. Obviously, the larger this value, the better the segmentation quality.

Figs. 4-6 display segmented results sampled from the $25 \mathrm{~K}$-means runs for each image. The images display typical results, i.e., results with classification accuracies as close to the average accuracy as possible. Often, this meant that an 
TABLE IV

Average Segmentation AcCuracy (Percentage) And All-Class IDENTIFICATION COUNT (AIC) USING K-MEANS (25 Runs) ACROSS ALL TESTS. NN: FEATURES Not NoRMALIZED. PCA: FEATURES REDUCED By PCA. FC: FEATURES REDUCED By FEATURE CONTRAST [7]

\begin{tabular}{c|c|c|c|c|c|c}
\hline & \multicolumn{2}{|c|}{ Bigun } & \multicolumn{2}{c|}{ Patch } & \multicolumn{2}{c}{ Star } \\
\hline Test & $\%$ & AIC & $\%$ & AIC & $\%$ & AIC \\
\hline$F_{G 18}$ & 75.8 & 3 & 74.1 & 0 & 80.2 & 0 \\
$F_{G 24}$ & 77.2 & 4 & 75.2 & 0 & 82.3 & 2 \\
$F_{C 24}$ & 57.4 & 0 & 82.5 & 14 & 89.5 & 24 \\
$F_{G 18}^{C 24}$ & 84.9 & 13 & 89.0 & 13 & 93.5 & 22 \\
$F_{G 24}^{C 24}$ & 83.9 & 11 & 90.0 & 15 & 93.6 & 22 \\
$F_{G 18} N N$ & 79.7 & 1 & 82.3 & 13 & 80.5 & 0 \\
$F_{G 24} N N$ & 87.2 & 14 & 79.7 & 12 & 83.5 & 3 \\
$F_{G 24}^{C 24} F C$ & 68.2 & 0 & 56.3 & 0 & 64.7 & 0 \\
\hline$F_{G 18}^{C 24} P C A$ & 83.5 & 11 & 90.3 & 16 & 89.2 & 15 \\
$F_{G 24}^{C 24} P C A$ & 81.0 & 7 & 88.0 & 15 & 88.8 & 14 \\
$F_{G 18}^{C 24} F C$ & 74.7 & 0 & 62.3 & 0 & 56.3 & 0 \\
& & & & & & \\
\hline
\end{tabular}

unsuccessful result is displayed and this is done to illustrate in what manner the segmentation tended to fail.

\section{Contrasting Discriminant and Segmentation Methods}

Segmentation procedures require the analysis of windows that can contain more than one texture, unlike the discriminant analysis that requires the use of pure texture samples. The windows with mixed texture types are expected to produce features that are a linear weighting of each of the textures in the image [23]. As a result, feature measures are produced that may mimic other textures in the image. This is especially true when a higher percentage of the image contains texture boundaries. The greater the degree of the texture boundaries, the greater the potential for segmentation error, and this is often reflected in the texture boundary being identified as a separate class. This is the reason that testing with bipartite images (an extreme case are bipartite images with two textures separated by a straight boundary down the middle) is inappropriate to truly evaluate the quality of an unsupervised texture segmentation approach.

\section{Hypothesis 3: Curse of Dimensionality}

This hypothesis states that the 48-D feature space generated by fusing the $F_{G 24}$ and $F_{C 24}$ feature sets will not lead to a decrease in segmentation accuracy compared to subfeature sets.

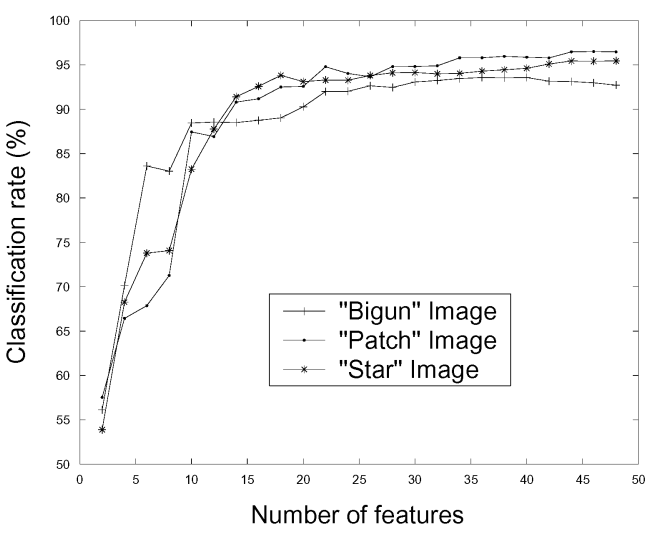

Fig. 3. Randomly selected texture features (from the set $F_{G 24}^{C 24}$ ) versus segmentation accuracy (percentage) for all three images.

This hypothesis asserts that there are sufficient feature vectors to properly represent each class's parameters in the 48-D feature space. Here, a simple test is used to illustrate the role of the curse of dimensionality for the proposed fused feature set.

Three curves (one for each image) are used to illustrate that the curse of dimensionality is generally not significant using the 48-D feature set (Fig. 3). The plots compare the number of texture features (horizontal axis) versus the classification accuracy (percentage) (vertical axis). Each curve is produced in the following fashion. Two features are randomly selected from the $F_{G 24}^{C 24}$ feature set. The K-means algorithm is run 25 times and the maximum classification accuracy produced is plotted. Then, another two features are randomly selected (of the remaining features) and an associated accuracy is plotted. This is continued until all 48 features are selected. Features are selected in an independent random order for each image.

The curse of dimensionality does not occur for either the Patch or Star images. For both of these images, the classification accuracy is monotonically increasing up to the full 48-D feature space. For the Bigun image, the classification accuracy decreases slightly only in the last few features, indicating that the maximum number of features has already been reached. This is possibly due to the Bigun image having seven classes and each of the classes, on average, are not well represented (in terms of total number of feature vectors) as for the Patch and Star classes. Effectively, the 48-D feature space is warranted without any detrimental effects.

Other authors are cautious about the curse of dimensionality with respect to image segmentation, but this caution has been historically derived from classification problems where individual textured samples are classified [16], [29], [30], [34]. In these cases, a restricted number of samples prevents the use of larger dimensional feature spaces. Image segmentation provides numerous feature vectors per class that allow for larger dimensional feature spaces. Also, the fused feature set $F_{G 24}^{C 24}$ is designed to fuse complementary texture features and other texture fusion papers have not considered such fusion in the past.

In a practical sense, for the case of image segmentation, there are many samples that can be used to accurately estimate class parameters. For example, consider the following "worst-case" scenario. Consider a single class represented by 2500 pixels (a small $50 \times 50$ pixel patch); then, given a 48 -D feature space, at 


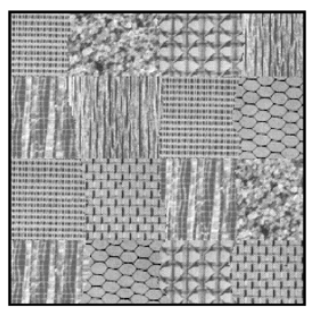

(a)

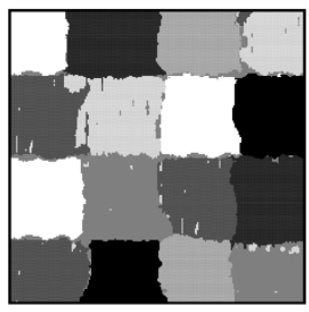

(f)

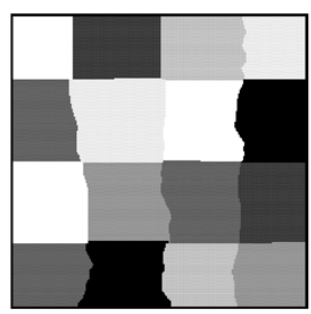

(b)

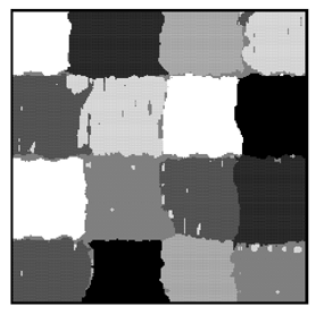

(g)

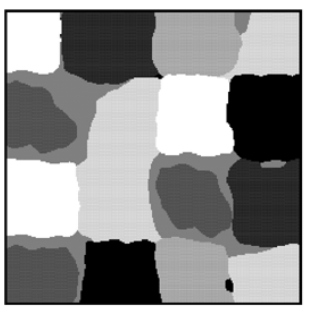

(c)

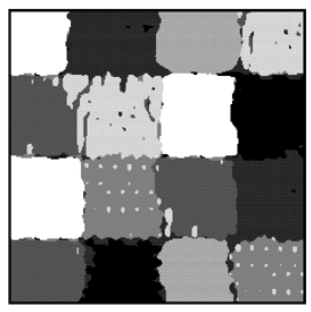

(h)

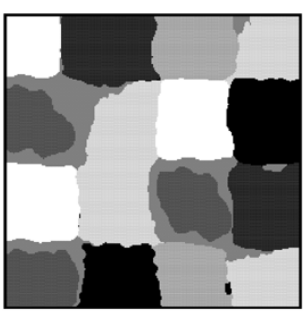

(d)

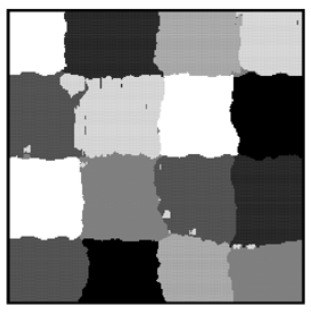

(i)

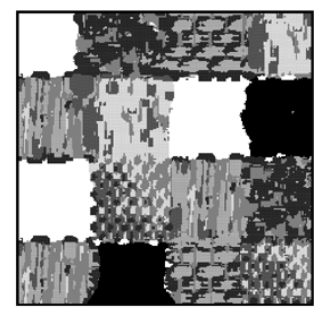

(e)

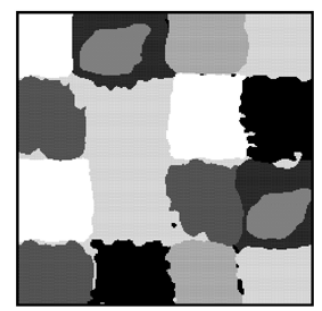

(j)

Fig. 4. (a) Original and (b) truth Bigun [33] images. Images (c)-(j) display typical segmentation results for various tests. (c) $F_{G 18}$. (d) $F_{G 24}$. (e) $F_{C 24}$. (f) $F_{G 18}^{C 24}$. (g) $F_{G 24}^{C 24}$. (h) $F_{G 24}$ NN. (i) $F_{G 24}^{C 24}$ PCA. (j) $F_{G 24}^{C 24}$ FC.

least 50 samples per feature space dimension are available for this class. With larger images and larger class spatial extents, additional feature vectors are available to estimate these parameters. Recall that Jain and Chandrasekaran [6] indicated a general guideline of using five to ten times as many samples as measurements. In the case of image segmentation, this is not a difficult threshold to achieve and the plots in Fig. 3 are in support. We expect that a higher number of samples per feature dimension are necessary for image segmentation. In actuality, the required number of samples per dimension will vary from image to image depending on the nature of the classes and the feature space relationship of all the classes. However, the curse of dimensionality is not expected to be a concern when performing texture image segmentation using proper feature extraction methods (such as $F_{G 24}^{C 24}$ ) and feature reduction methods should not be required. A simple test (as provided in Fig. 3) can be performed to verify this conjecture for a given dataset.

\section{E. Hypothesis 4: Comparing Nonfused and Fused Feature Sets}

This hypothesis states that the fused feature sets will achieve higher segmentation accuracies than any of the nonfused feature sets. Here, Hypothesis 4 will focus on the results in the first five rows of Table IV, i.e., those results pertaining to each case presented in Table I. The fused feature sets have higher segmentation accuracies relative to the nonfused feature sets. For example, the Star image feature set $F_{G 18}$ improves from $80.2 \%$ [Fig. 6(c)] to $93.5 \%$ [Fig. 6(f)] when fused with $F_{C 24}$ and $F_{G 24}$ improves from $82.3 \%$ [Fig. 6(d)] to $93.6 \%$ [Fig. $6(\mathrm{~g})$ ] when fused with $F_{C 24}$. The AIC values (defined in Section V-B) agree with the percentage results. The independent feature sets are able to uniquely identify all of the classes in each image fewer than five times using $F_{G 18}$ and $F_{G 24}$. After fusion with the $F_{C 24}$ feature set, the AIC values end up in the range of 11 to 22 , a dramatic increase in the segmentation ability. In summary, the fused feature sets are strongly advocated over using the individual feature sets.

\section{F. Hypothesis 5: Segmentation With Image Noise}

This hypothesis states that in the presence of noise, the fused feature set will achieve higher segmentation accuracies than using Gabor filters alone. This relates back to Figs. 1 and 2 where Gabor filters had poorer texture feature representation in the presence of increasing noise. When fused with GLCP features, it is expected that features more robust to point noise will be produced. To test this hypothesis, each image was segmented using $F_{G 24}$ and $F_{G 24}^{C 24}$ feature sets with increasing point Gaussian noise added to the image. Each noise level used 25 K-means runs for each feature set. Fig. 7 presents the three plots, one for each image. In each case, the fused feature set typically maintained a higher classification rate relative to the pure feature set. Of notice with the Bigun image [Fig. 7(a)] is the trend for the fused feature set to improve relative to the pure feature set as the noise magnitude is increased. In summary, the hypothesis is supported since the fused feature set outperforms the nonfused feature set in the presence of noise.

\section{G. Hypothesis 6: Normalization of Gabor Filters}

This hypothesis states that the linear normalization of Gabor filtered texture features will reduce their segmentation ability. Papers that use Gabor filters for texture analysis generally use some form of normalization or scaling of these features prior to classification [2], [3], [7]. In Section V-B, the use of normalization prior to the application of a clustering methods such as K-means is acknowledged. However, it is hypothesized that normalization is not necessary when using only Gabor filtered magnitude features since each Gabor feature dimension has the same unit. As a result, the relative strength of the Gabor filter magnitude response across different filters is considered important for texture identification and normalization is expected to remove this information. However, for different GLCP statistics and the combination of GLCP and Gabor features, normalization is necessary, since the units used in GLCP are different across different GLCP statistics. 


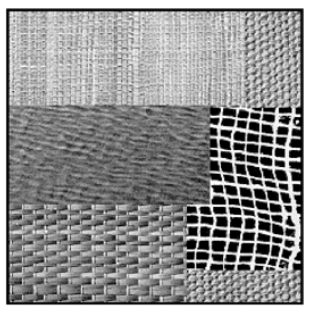

(a)

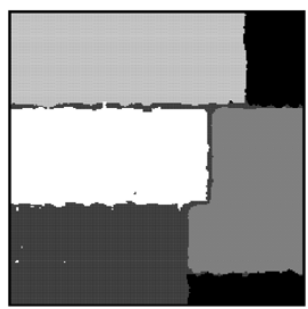

(f)

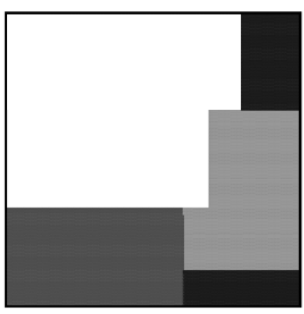

(b)

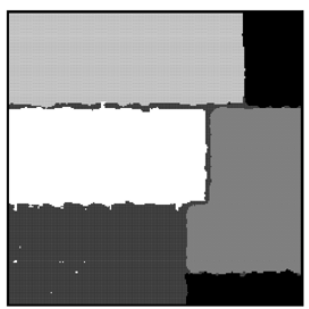

(g)

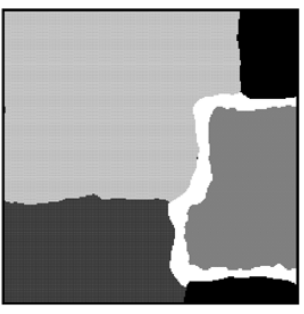

(c)

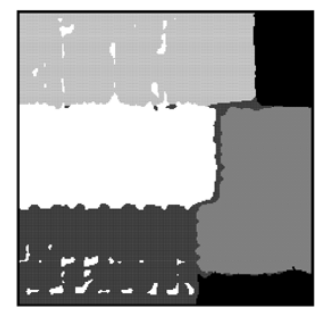

(h)

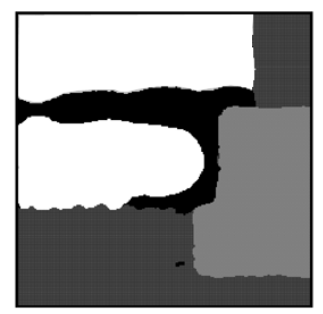

(d)

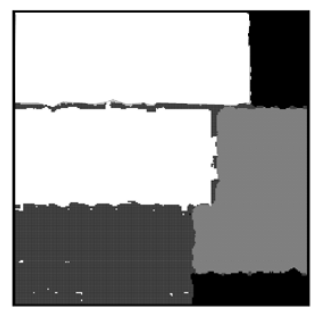

(i)

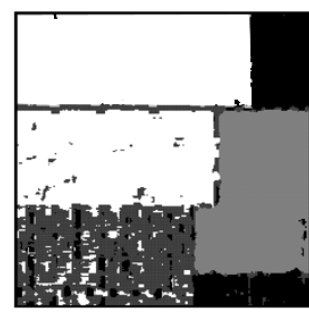

(e)

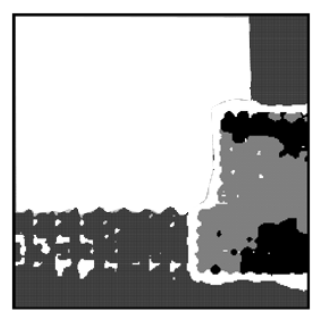

(j)

Fig. 5. (a) Original and (b) truth Patch images. Images (c)-(j) display typical segmentation results for various tests. (c) $F_{G 18}$. (d) $F_{G 24}$. (e) $F_{C 24}$. (f) $F_{G 18}^{C 24}$. (g) $F_{G 24}^{C 24}$. (h) $F_{G 24}$ NN. (i) $F_{G 24}^{C 24}$ PCA. (j) $F_{G 24}^{C 24}$ FC.

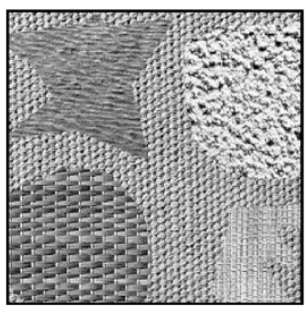

(a)

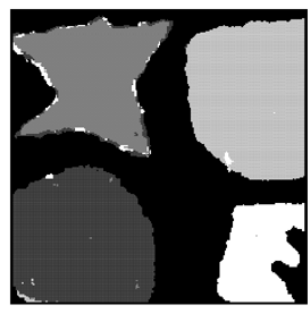

(f)

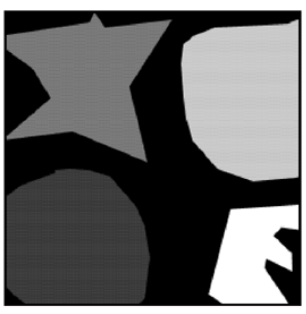

(b)

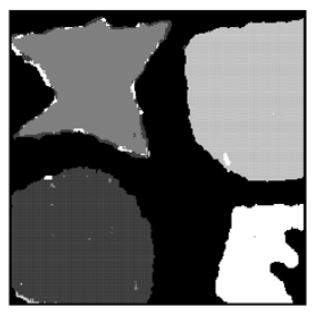

(g)

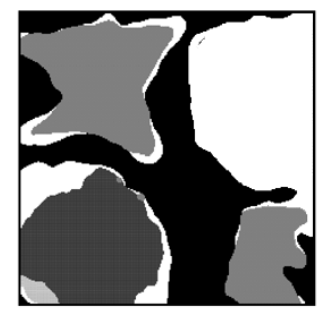

(c)

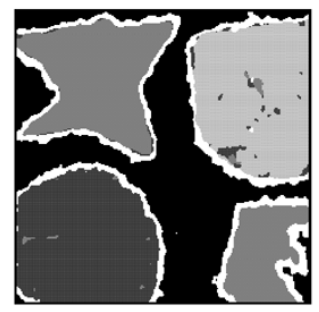

(h)

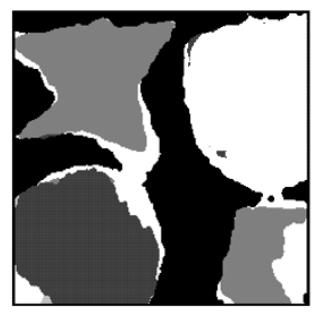

(d)

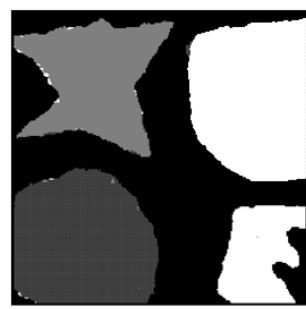

(i)

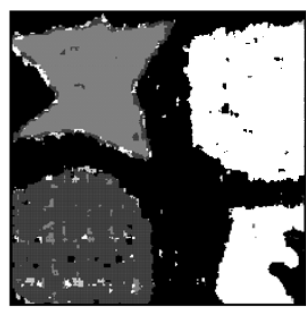

(e)

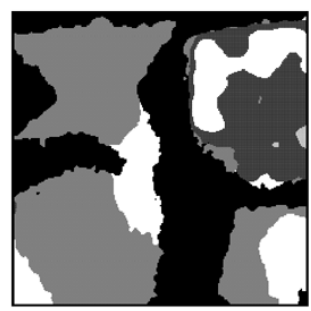

(j)

Fig. 6. (a) Original and (b) truth Star images. Images (c)-(j) display typical segmentation results for various tests. (c) $F_{G 18}$. (d) $F_{G 24}$. (e) $F_{C 24}$. (f) $F_{G 18}^{C 24}$. (g) $F_{G 24}^{C 24}$. (h) $F_{G 24}$ NN. (i) $F_{G 24}^{C 24}$ PCA, (j) $F_{G 24}^{C 24}$ FC.

The impact of normalization on each pure Gabor filter set is investigated. Table IV includes the segmentation accuracies across all three images for normalized and nonnormalized (indicated by NN) Gabor magnitude features. In every case, the segmentation accuracies for the nonnormalized Gabor filtered features are higher than the normalized cases. For most cases, the $F_{G 24}$ feature set has a strong increase in the AIC values, e.g., for the Bigun image the AIC value increases from 4 to 14 and for the Patch image the AIC value increases from 0 to 12. Note that, paradoxically, the linear normalization does not modify the Fisher criterions (i.e., the weighted feature space separation is not modified under linear transformations); however, the same linear normalization is detrimental to the segmentation accuracy. For the segmented images (Figs. 4-6), it is noted that the normalized cases [(c) and (d)] have tremendous difficulties segmenting the textural boundaries accurately, although, generally within each texture region the segmentation is accurate. It is also noted that for the Bigun image, the nonnormalized Gabor filters performed better than every other test, although this was not true for the Patch and Star images.

\section{H. Hypothesis 7: Feature Reduction}

This hypothesis states that the feature reduction of fused texture feature sets will lead to poorer segmentation ability. Two techniques have been presented in Section II to perform the reduction: the commonly used PCA and the more recently introduced FC method [7]. The same methodology for PCA and FC that was used for the results in Table III is used for the results in Table IV.

Table IV indicates the accuracies of the two fused feature sets and their reduction using PCA and FC. PCA generally produces segmentation accuracies similar to the full feature 


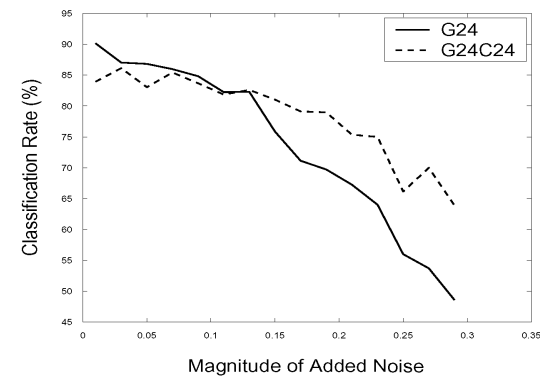

(a)

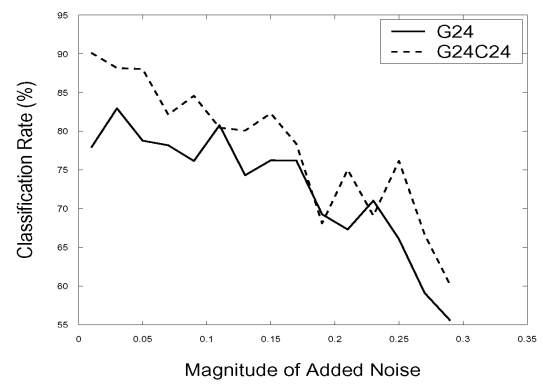

(b)

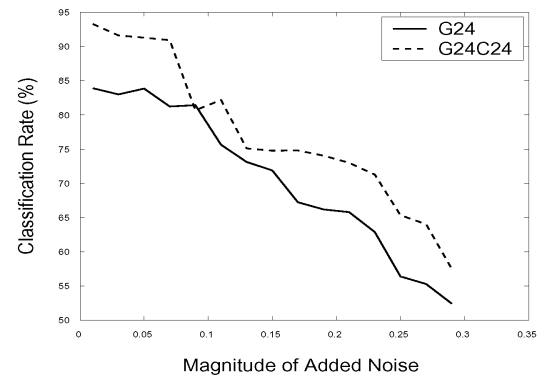

(c)

Fig. 7. Average classification rate (percentage) (over 25 K-means runs per noise level) with respect to the magnitude of added noise. (a) Bigun image. (b) Patch image. (c) Star image.

set. The accuracies for the Bigun and Patch images follow this trend; however, the Star image has a noticeable decrease in the percentage accuracy using the PCA reduced feature set compared to using the full feature set. Overall, the PCA method produces effective segmentation results that marginally weaker than using the full feature set. Bigun [9] felt that the PCA transform was inappropriate for feature reduction prior to clustering due to undesired interaction between classes. However, the results here indicate that the PCA transform is quite adept at maintaining the class distinctions while reducing the feature space dimensionality. The AIC values and the results indicated in the segmented images [see image (i) in Figs. 4-6 for the $F_{G 24}^{C 24}$ PCA result] support the percentage accuracy results. The number of principal components retained is image dependent. For the $F_{G 24}^{C 24}$ and $F_{G 18}^{C 24}$ feature sets, the Bigun image retained 28 and 23 components, the Patch image retained 25 and 21 components, and the Star image retained 36 and 31 components. That the Star image retained a significantly higher number of components relative to the other two images is perhaps indicative of the feature space variability of its classes, making it a more difficult image to segment.

The FC method, on the other hand, is detrimental to the segmentation accuracy across all three images. For example, for the Star image, $F_{G 18}^{C 24}$ and $F_{G 24}^{C 24}$ produce accuracies of $93.5 \%$ and $93.6 \%$, respectively, and their FC counterparts only manage accuracies of $67.3 \%$ and $64.7 \%$. The Bigun and Patch image generate similar reductions in segmentation accuracy. No FC reduction properly identifies each class in any image and so the AIC values are all zero. As a result, the FC method is not advocated for feature space reduction prior to unsupervised segmentation. Image segmentations using FC depict boundary and textured region distortions [see (j) in each of Figs. 4-6].

Separability measures are used to support the percentage accuracies and AIC results. Table V displays the average class-pairwise separability across all three images for $F_{G 18}^{C 24}$ and $F_{G 24}^{C 24}$ and the corresponding feature reductions (PCA and FC). The separability is measured using feature vectors assigned to the ground truth classes (as opposed to the segmented result). This reflects the true separability of the derived texture features. These results mirror those generated by percentage accuracies and AIC. PCA is able to retain approximately $90 \%$ of the separability of the original feature sets. However, FC reduces the separability to approximately $70 \%$ of the original feature sets.
TABLE $\mathrm{V}$

Class Feature Space Separability for All Six Texture Feature Sets By AVERAGE Class-PAIRWISE Fisher LINEAR CRITERION VALUES

\begin{tabular}{c|c|c|c}
\hline Feature Set & Bigun & Patch & Star \\
\hline$F_{G 24}^{C 24}$ & 31.50 & 64.50 & 28.47 \\
$F_{G 24}^{C 24}$ by PCA (98\%) & 27.91 & 54.65 & 25.98 \\
$F_{G 24}^{C 24}$ by FC (5\%) & 24.79 & 34.54 & 15.78 \\
$F_{G 18}^{C 24}$ & 30.37 & 63.41 & 27.14 \\
$F_{G 18}^{C 24}$ by PCA (98\%) & 26.79 & 53.65 & 24.93 \\
$F_{G 18}^{C 24}$ by FC (5\%) & 23.89 & 33.86 & 15.45 \\
\hline
\end{tabular}

Again, the FC method is not as effective as PCA for retaining texture feature content.

There are a few suggestions to explain the weaker performance of the FC method. The FC method was originally created as a detection method for homogeneous texture regions [35]. Under the circumstances where the feature space contains multiple classes, perhaps the FC method is not designed to properly enhance the features for a given class, since the enhancement will actually be applied based on the interpretation of multiple classes along a feature dimension. For example, the maximum value $\max \left(f_{i}\right)$ is representative of the maximum feature value of one class, but other distributions (representing other classes) will be apparent in the feature dimension. Also, the mean value mean $\left(f_{i}\right)$ is a mean of all of the class contributions, not based on a single class. The inclusion of multiple classes is expected to limit the FC method for feature reduction. Also, the FC method is based on the identification of strong feature responses (by accessing the maximum feature value) and is not designed to identify weak consistent magnitude responses (textures containing low contrast sinusoids textures will cause lower but consistent magnitude responses). This information, critical to detection of some textures, could be masked in the FC method.

The implementation used by Pichler et al. has one distinction. With respect to Gabor filters, it is advocated to use the magnitude response as opposed to the response in power [25]; however, Pichler et al. and consequently the reported results in this paper use the power coefficients. The tests were also run using 
only the magnitude response. These magnitude response results (not presented) demonstrated an improvement over the power coefficients, but were still poorer in all cases relative to the PCA results.

In summary, neither the PCA nor the FC method is advocated to improve the segmentation accuracy. PCA feature reduction can be advocated to improve the computational demands of the clustering procedure, but the quality of the segmentation is expected to remain the same or to be lowered, given an appropriate feature set. Neither method is recognized to enhance the separability of the clusters in the feature space.

\section{CONCLUSION}

The paper produces a number of significant contributions. In summary:

1) A design-based method to fuse Gabor filter texture features and co-occurrence probability features is described and implemented. Whereas other research papers generally fuse features blindly, a rationale is provided here for the fusion of these particular features. The fusion is based on the theoretical analysis and experimental verification of each method so as to combine robust, reliable, and complementary features.

2) Discriminant analysis indicates the following.

- The fused texture features generate improved separability relative to using the individual feature sets.

- Feature reduction (using PCA or FC) dramatically decreases the class separability of the feature sets.

3) The curse of dimensionality does not affect the performance of the segmentation accuracy, given the appropriate feature set. Larger images with texture classes having larger spatial extents will only improve this situation.

4) Fused feature sets $\left(F_{G 24}^{C 24}\right.$ and $\left.F_{G 18}^{C 24}\right)$ consistently outperform the independent feature sets $\left(F_{G 24}, F_{G 18}\right.$, and $\left.F_{C 24}\right)$ in segmentation accuracy, both for noise-free and noisy images.

5) Linear normalization of Gabor filtered features removes discriminating information and reduces the capability of these features to perform segmentation accurately.

6) Feature reduction applied to an appropriate feature set is not expected to improve the segmentation accuracy. More specifically, we have the following.

- Feature reduction using PCA is expected to maintain or reduce the segmentation accuracy.

- Feature reduction using FC is expected to severely reduce the segmentation accuracy.

\section{ACKNOWLEDGMENT}

The authors would like thank the reviewers for their contributing comments.

\section{REFERENCES}

[1] D. A. Clausi, "K-means iterative Fisher (KIF) unsupervised clustering algorithm applied to image texture segmentation," Pattern Recognit., vol. 35, no. 9, pp. 1959-1972, 2002.

[2] T. Randen and J. H. Husoy, "Filtering for texture classification: A comparative study," IEEE Trans. Pattern Anal. Mach. Intell., vol. 21, no. 4, pp. 291-310, Apr. 1999.
[3] A. K. Jain and F. Farrokhnia, "Unsupervised texture segmentation using Gabor filters," Pattern Recognit., vol. 24, no. 12, pp. 1167-1186, 1991.

[4] D. Clausi and H. Deng, "Unsupervised segmentation of texture images through optimal fusion of Gabor and co-occurrence texture features," in Proc. 16th Int. Conf. Vision Interface, Halifax, NS, Canada, Jun. 2003, pp. 237-242.

[5] M. Tuceryan and A. K. Jain, Handbook of Pattern Recognition and Computer Vision, Chapter 2: Texture Analysis, Singapore: World Scientific, 1993.

[6] A. Jain and B. Chandrasekaran, Dimensionality and Sample Size Considerations in Pattern Recognition Practice. Amsterdam, The Netherlands: North-Holland, 1982, vol. 2, pp. 835-855.

[7] O. Pichler, A. Teuner, and B. J. Hosticka, "An unsupervised texture segmentation algorithm with feature space reduction and knowledge feedback," IEEE Trans. Image Process., vol. 7, no. 1, pp. 53-61, Jan. 1998.

[8] A. H. S. Solberg and A. K. Jain, "Texture fusion and feature selection applied to SAR imagery," IEEE Trans. Geosci. Remote Sens., vol. 35, no. 2, pp. 475-479, Mar. 1997.

[9] J. Bigun, "Unsupervised feature reduction in image segmentation by local transforms," Pattern Recognit. Lett., vol. 14, pp. 573-583, 1993.

[10] C. Liu and H. Wechsler, "A shape- and texture-based enhanced Fisher classifier for face recognition," IEEE Trans. Image Process., vol. 10, no. 4, pp. 598-608, Apr. 2001.

[11] R. Manduchi, "Bayesian fusion of color and texture segmentations," in Proc. IEEE Int. Conf. Computer Vision, vol. 2, 1999, pp. 956-963.

[12] K. M. Bashar and N. Ohnishi, "Fusing cortex transform and intensity based features for image texture classification," in Proc. 5th Int. Conf. Information Fusion, Jul. 2002, pp. 1463-1469.

[13] S. Chindaro, K. Sirlantzis, and F. Deravi, "Color space fusion for texture recognition," in Proc. 4th EURASIP Conf. Video/Image Processing and Multimedia Communications, Jul. 2003, pp. 181-186.

[14] G. Trianni, M. Tosi, F. Dell'Acqua, P. Gamba, and G. Lisini, "Fusion of texture measures for urban area characterization," in Proc. 7th Int. Conf. Information Fusion, Jun./Jul., 2004, pp. 991-998.

[15] D. Zongker and A. Jain, "Algorithms for feature selection: An evaluation," in Proc. Int. Conf. Pattern Recognition, vol. 2, Aug. 1996, pp. $18-22$.

[16] D. A. Clausi, "Comparison and fusion of co-occurrence, Gabor and MRF texture features for classification of SAR sea-ice imagery,' Atmos.-Ocean, vol. 39, no. 3, pp. 183-194, 2000.

[17] R. Duda, P. Hart, and D. Stork, Pattern Classification, 2nd ed. New York: Wiley, 2001.

[18] J. Mao and A. Jain, "Texture classification and segmentation using multiresolution simultaneous autoregressive models," Pattern Recognit. vol. 25, no. 2, pp. 173-188, 1992.

[19] D. Weszka, C. R. Dyer, and A. Rosenfeld, "A comparative study of texture measures for terrain classification," IEEE Trans. Syst., Man, Cybern., vol. SMC-6, no. 4, pp. 269-285, Apr. 1976.

[20] R. W. Conners and C. A. Harlow, "A theoretical comparison of texture algorithms," IEEE Trans. Pattern Anal. Mach. Intell., vol. PAMI-2, no. 5, pp. 204-221, May 1980.

[21] P. Ohanian and R. Dubes, "Performance evaluation for four classes of textural features," Pattern Recognit., vol. 25, no. 8, pp. 819-833, 1992.

[22] D. G. Barber, M. E. Shokr, R. A. Soulis, and D. G. Flett, "A comparison of second-order classifiers for SAR sea ice discrimination," Photogramm. Eng. Remote Sens., vol. 59, no. 9, pp. 1397-1408, 1993.

[23] D. Clausi and B. Yue, "Comparing co-occurrence probabilities and Markov random fields for texture analysis," IEEE Trans. Geosci. Remote Sens., vol. 42, no. 1, pp. 215-228, Jan. 2004.

[24] J. Du Buf, M. Kardan, and M. Spann, "Texture feature performance for image segmentation," Pattern Recognit., vol. 23, no. 3/4, pp. 291-309, 1990.

[25] D. A. Clausi and M. E. Jernigan, "Designing Gabor filters for optimal texture separability," Pattern Recognit., vol. 33, pp. 1835-1849, 2000.

[26] A. C. Bovik, M. Clark, and W. S. Geisler, "Multichannel texture analysis using localized spatial filters," IEEE Trans. Pattern Anal. Mach. Intell., vol. 12, no. 1, pp. 55-73, Jan. 1990.

[27] R. M. Haralick, K. Shanmugam, and I. Dinstein, "Textural features for image classification," IEEE Trans. Syst. Man. Cybern., vol. SMC-3, no. 6, pp. 610-621, Jun. 1973.

[28] D. A. Clausi, "An analysis of co-occurrence texture statistics as a function of grey level quantization," Can. J. Remote Sens., vol. 28, no. 1, pp $1-18,2002$.

[29] L. K. Soh and C. Tsatsoulis, "Texture analysis of SAR sea ice imagery using gray level co-occurrence matrices," IEEE Trans. Geosci. Remote Sens., vol. 37, no. 2, pp. 780-795, Mar. 1999.

[30] M. E. Shokr, "Evaluation of second-order texture parameters for sea ice classification from radar images," J. Geophys. Res., vol. 96, no. C6, pp. $10625-10640,1991$. 
[31] S. Zucker and D. Terzopoulos, "Finding structure in co-occurrence matrices for texture analysis," Comput. Graph. Image Process., vol. 12, pp. 286-308, 1980.

[32] P. Brodatz, Texture-A Photographic Album for Artists and Designers. New York: Reinhold, 1968.

[33] J. Bigun and J. M. du Buf, "N-folded symmetries by complex moments in Gabor space and their applications to unsupervised texture segmentation," IEEE Trans. Pattern Anal. Mach. Intell., vol. 16, no. 1, pp. 80-87, Jan. 1994.

[34] D. G. Barber and E. F. LeDrew, "SAR sea ice discrimination using texture statistics: A multivariate approach," Photogramm. Eng. Remote Sens., vol. 57, no. 4, pp. 385-395, 1991

[35] A. Teuner, O. Pichler, and B. Hosticka, "Unsupervised texture segmentation of images using tuned matched Gabor filters," IEEE Trans. Image Process., vol. 4, no. 6, pp. 863-870, Jun. 1995.

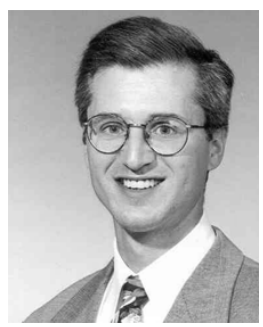

David A. Clausi received the B.A.Sc., M.A.Sc., and $\mathrm{Ph} . \mathrm{D}$. degrees in systems design engineering from the University of Waterloo, Waterloo, ON, Canada, in 1990, 1992, and 1996, respectively.

After completing the Ph.D. degree, he worked in the medical imaging field at Mitra Imaging, Inc., Waterloo. He started his academic career in 1997 as an Assistant Professor of geomatics engineering at the University of Calgary, Calgary, AB, Canada. In 1999, he returned to his alma mater as an Assistant Professor of systems design engineering and was awarded tenure and a promotion to Associate Professor in 2003. He is an active interdisciplinary and multidisciplinary researcher. He has a prestigious publication record, publishing refereed journal and conference papers in the diverse fields of remote sensing, computer vision, algorithm design, and biomechanics. His primary research interest is the automated interpretation of SAR sea ice imagery, in support of operational activities of the Canadian Ice Service. The research results have led to successfully commercial implementations.

Dr. Clausi has received numerous graduate scholarships, conference paper awards, and a Teaching Excellence Award.

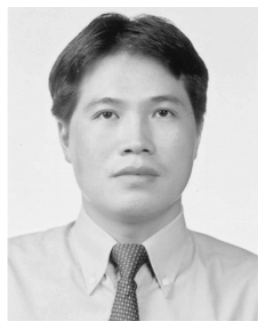

Huang Deng received the B.A.Sc. degree from Beijing University, Beijing, China, in 1992, the M.Eng. degree from the Research Institute of Petroleum Exploration and Development, China, in 1995, and the $\mathrm{Ph} . \mathrm{D}$. degree from Nanyang Technological University, Singapore, in 2001.

From 2000 to 2002, he was an Information Technology Analyst for a regional bank in Singapore. From 2002 to 2004, he was a Postdoctoral Research Fellow in systems design engineering at the University of Waterloo, Waterloo, ON, Canada. He is currently with PCI Geomatics in Hull, QC, Canada. His research interests are in image processing (texture modeling, image classification, image segmentation, and image retrieval), computer vision, pattern recognition, and remote-sensing data analysis. 